Original Article

\title{
Clinical application of circuit training for subacute stroke patients: a preliminary study
}

\author{
Sun Mi Kim, MD²), Eun Young Han, MD²*, Bo Ryun Kim, MD, PhD²), Chul Woong Hyun ${ }^{2)}$ \\ 1) Department of Rehabilitation Medicine, Dongtan Sacred Heart Hospital, Hallym University College \\ of Medicine, Republic of Korea \\ 2) Department of Rehabilitation Medicine, Jeju National University Hospital, Jeju National University \\ School of Medicine, Republic of Korea
}

\begin{abstract}
Purpose] To investigate how task-oriented circuit training for the recovery motor control of the lowerextremity, balance and walking endurance could be clinically applied to subacute stroke inpatient group therapy. [Subjects and Methods] Twenty subacute stroke patients were randomly assigned to the intervention group $(\mathrm{n}=10)$ or the control group $(\mathrm{n}=10)$. The intervention consisted of a structured, progressive, inpatient circuit training program focused on mobility and gait training as well as physical fitness training that was performed for 90 minutes, 5 days a week for 4 weeks. The control group received individual physiotherapy of neurodevelopmental treatment for 60 minutes, 5 days a week for 4 weeks. Outcome measures were lower-extremity motor control, balance, gait endurance and activities of daily living before and after 4 weeks. [Results] There were no significant differences at baseline between the two groups. After 4 weeks, both groups showed significant improvements in all outcome measures, but there were no significant differences between the two groups during the invention period. [Conclusion] In spite of the small sample size, these findings suggest that task-oriented circuit training might be used as a cost-effective and alternative method of individual physiotherapy for the motor recovery of lower-extremity, balance and walking endurance of subacute stroke patients.

Key words: Stroke, Rehabilitation, Circuit training
\end{abstract}

(This article was submitted Sep. 14, 2015, and was accepted Oct. 14, 2015)

\section{INTRODUCTION}

Stroke is a common, serious, and disabling health-care problem throughout the world ${ }^{1}$. Intensive rehabilitation therapy in the subacute phase of stroke recovery is essential for recovery of functional ability ${ }^{2}$. In Korea, most patients want to receive intensive conventional rehabilitation therapy, based on neurodevelopmental treatment (NDT), but intensive rehabilitation therapy is time-limited and requires the presence of one therapist per patient. Thus, it is only possible in a rehabilitation inpatient setting due to the limited rehabilitation service resources of service providers in the Korean medical insurance system. On the other hand, circuit training that is composed of individual programs that focus on functional tasks can be provided in a group setting of two or more patients by a physiotherapist and is potentially cost saving, because it involves a low therapist-to-patient ratio, compared with conventional physiotherapy ${ }^{3)}$.

There is growing evidence that circuit training is effective at improving the walking competency of patients in the chronic phase of stroke ${ }^{4}$. Although only one study has demonstrated the effectiveness of circuit training as an alternative to individual physiotherapy for stroke patients receiving inpatient rehabilitation ${ }^{5}$, few studies have researched how to conduct circuit training for patients in the subacute stage of stroke in inpatient rehabilitation facilities.

Therefore, the purpose of this study was to determine the feasibility of circuit training during inpatient rehabilitation in this country, and investigate whether a 90 -min task-oriented circuit training program that concentrates on improving mobility

\footnotetext{
*Corresponding author. Eun Young Han (E-mail: clearblue10@naver.com)

(C)2016 The Society of Physical Therapy Science. Published by IPEC Inc.

This is an open-access article distributed under the terms of the Creative Commons Attribution Non-Commercial No Derivatives (by-nc-nd) License $<$ http://creativecommons.org/licenses/by-nc-nd/3.0/>.
} 
and gait training is effective for the recovery of lower-extremity motor control, balance and walking endurance and how it can be conducted for subacute phase of stroke patients.

\section{SUBJECTS AND METHODS}

Twenty patients (13 males and 7 females; mean age, $65.6 \pm 9.2$ years) with subacute stroke (i.e., within 3 months of stroke) were selected for this study. Patients were recruited from the Department of Physical Medicine and Rehabilitation of Jeju National University Hospital between August 2012 and October 2013. All the patients had first-ever cerebral stroke that involved the cortical or subcortical area. Lesions were confirmed through magnetic resonance imaging or computed tomography. The eligibility criteria were: a clinical diagnosis of a first stroke confirmed by neuroimaging (computed tomography or magnetic resonance imaging); a hemiparesis; a time interval between stroke and recruitment of 3 months or less; the ability to comprehend the instructions for the testing procedures; and mild to moderate walking deficit, as indicated by Functional Ambulation Category (FAC) between 3 and 46,7).

Exclusion criteria were as follows: a severe cognitive impairment (K-MMSE $\leq 10)$ or aphasia; previous stroke history; not independent 'sit to stand' activity (Berg Balance Scale score $<18$ ) orthopedic condition or pain that limited participation in exercise; and visual impairment or vestibular system deficit that caused balance impairment.

After subjects passed the screening criteria, they provided their informed consent to participation in this study. The study protocol was reviewed and approved by the Institutional Review Board of Jeju National University Hospital.

This was a randomized, single-blind study. Participants were randomly allocated in a 1:1 ratio to the two study groups: 10 patients to the circuit training group and another 10 patients to the control group. The randomization was performed using a sealed envelope technique. The examiner was blinded as to whether the participants were in the experimental group or control group. Multidisciplinary rehabilitation programs except for physiotherapy were provided equally to both groups per usual practice.

The lower limb score from the Fugl-Meyer assessment (FMA-LL) was used to evaluate the motor function of both lower extremities including the hip, knee and ankle. The FMA-LL is widely used for comprehensive clinical examination of leg motor function, and the maximal score is 34 points ${ }^{9}$.

The 6 min walk test (6MWT) was used as a measure of gait endurance. Subjects performed the 6 min walk test, in which they were instructed to cover as much distance as possible in $6 \mathrm{~min}^{10)}$. Subjects walked around a circuit marked in a $50 \mathrm{~m}$ hallway and were allowed to stop and rest if necessary.

Balance function was evaluated using the Berg Balance Scale, which measures balance while performing a variety of functional tasks that are common in everyday life ${ }^{8,11}$. Each task is rated on a five-point scale (range, 0-4). The maximum score is 56, and high scores indicate better balance. The tasks progress from sitting to comfortable standing, tandem standing, and single leg standing, and assess both static and dynamic balance by using tasks that involve reaching and weight transfer.

The Korean version of the Modified Barthel Index was used to evaluate activities of daily living (ADL) function ${ }^{12)}$. The Korean version of the Modified Barthel Index is a reliable, valid tool for measuring the ADL of patients in Korea and has a maximal score of 100 indicating complete independence in ADL.

All evaluations were conducted at baseline and repeated after completion of the 4 week intervention.

In the circuit training group, participants participated in 90-min circuit-training classes, 5 times per week for 4 weeks. At least two patients under the supervision of one physiotherapist attended to all the classes of circuit training. Circuit training consisted of a 5-min warm-up period, five classes of 15-min duration of complex exercises interspersed by 1-min rest, and a 5-min cool-down period. The total duration of the structured protocol was, therefore, $90 \mathrm{~min}$. All instructions were standardized and provided to the patients using multimedia and instruction (video and color instruction guideline brochure) by a physical therapist.

There were five categories of complex exercises including trunk exercise and active sitting practice, sit-to-stand practice, standing and walking practice, aerobic exercise training and strengthening training.

Categories 1-3 were core activities including mobility-related tasks that address key impairments and functional limitations, similar to the study of Dean et al. ${ }^{3)}$, and categories 4 and 5 were physical fitness training activities. The core activities were active sitting (easiest; category 1), sit-to-stand from chairs of various heights (more difficult; category 2), and standing and walking (most difficult; category 3).The core activities were individually adapted for each patient according to their functional levels and progress, such that the level of complexity was appropriate for the ability of the patient. Participants' progress was continuously monitored. When a patient completed an easier stage (category 1 or 2), or showed increased level of walking independence during the intervention, they were permitted to move up to the next category of core activities in the next 15 min exercise period. However, if a patient could not perform the activities in a category by himself, that patient was not permitted to attempt the next category of the core activity and was instructed to repeat the previous category. For example, until a patient was able to walk overground with minimal assistance, they did not attempt the exercises in category 3 , and performed only categories 1 and 2 . The core activities were performed in the first three 15 -min periods of the circuit training class after warm-up.

The physical fitness training activities were aerobic exercise training (category 4; performed in the fourth 15 min period 
of the circuit training class) and strengthening training (category 5; performed in the fifth 15 min period). Aerobic exercise was performed on a treadmill and workload was increased by increasing treadmill speed or adding an inclination. When a patient could not perform a treadmill activity safely, a stationary bike was used and the workload was increased by increasing bicycle resistance.

For strengthening training, participants performed the following targeted movements: hip abduction/flexion, knee flexion/ extension and ankle dorsiflexion. A Thera-Band ${ }^{\circledR}$ (Hygenic Corporation, Akron, Ohio, USA) was used to provide resistance, and participants performed two sets of $8-15$ repetitions of each exercise. Progression was achieved by increasing the number of repetitions or load according to subjective tolerance.

Participants in the control group received conventional individual physiotherapy for 30 min twice a day (total 60 min), 5 days a week for the 4 weeks. The content of the individual physiotherapy sessions was based on neurodevelopmental treatment for motor recovery ${ }^{13)}$.

For Statistical analysis, descriptive statistics were used to calculate means and standard deviations (SD). Wilcoxon's signed-rank test was used to compare the baseline values between two groups. The change in each outcome measure from pre- to post-treatment was evaluated separately in each group using the Wilcoxon signed rank test. The change in each outcome measure from pre- to post-treatment was compared between the two groups using a Mann-Whitney test. A p value less than 0.05 was considered significant. Statistical analyses were performed using SPSS for Windows version 20 (IBM-SPSS, Inc., Chicago, IL, USA).

\section{RESULTS}

The baseline demographic characteristics of both groups are presented in Table 1. No statistically significant differences between the two groups were found at baseline. Baseline FMA-LL, BBS and 6MWT distance are listed in Table 1 and show no significant intergroup-differences. Both types of treatment were well-tolerated with an attendance rate of $100 \%$, and all participants completed the study. There were no serious adverse events during the study period.

Clinical characteristics including lower-extremity motor control (FMA-LL), balance (BBS) and gait endurance (6MWT distance) before and after the intervention are described in Table 2. Both groups had significant improvement from pre-to post-treatment in all outcome measures (Table 2). However, there was no significant difference in the change in all parameters from pre- to post-intervention between the two groups (Table 3).

\section{DISCUSSION}

Our findings demonstrate that task-oriented circuit training for 90 minutes was as effective as individual physiotherapy (NDT) for 60 minutes for functional recovery in terms of lower-extremity motor control, balance, walking endurance and

Table 1. Baseline demographic and clinical characteristics of the participants

\begin{tabular}{lcc}
\hline & Circuit training group & Control group \\
\hline Numbers & 10 & 10 \\
Age (years) & $65.2 \pm 10.1$ & $66.0 \pm 8.8$ \\
Gender (male) & 6 & 7 \\
Height (cm) & $165.0 \pm 9.7$ & $166.1 \pm 7.8$ \\
Weight (kg) & $63.7 \pm 11.8$ & $67.9 \pm 9.5$ \\
Body mass index & $23.2 \pm 2.1$ & $24.5 \pm 2.4$ \\
Stroke type & & \\
$\quad$ Ischemic & 8 & 8 \\
Hemorrhagic & 2 & 2 \\
Affected side & & \\
$\quad$ Left & 6 & 5 \\
$\quad$ Right & 4 & 5 \\
Stroke duration (days) & $30.1 \pm 21.8$ & $29.9 \pm 20.3$ \\
FMA-LL & $25.4 \pm 7.5$ & $22.9 \pm 7.9$ \\
BBS & $36.3 \pm 11.7$ & $39.0 \pm 8.7$ \\
6MWT, m & $167.5 \pm 121.8$ & $157.5 \pm 64.0$ \\
K-MBI & $65.7 \pm 23.3$ & $57.4 \pm 22.4$ \\
\hline
\end{tabular}

Values are numbers or mean \pm standard deviation.

FMA-LL: paretic lower limb score of the Fugl-Meyer assessment; BBS: Berg balance test; 6MWT: 6-minute walk test; K-MBI: Korean version of the Modified Barthel Index 
Table 2. Clinical outcome measures before and after treatment

\begin{tabular}{lcccc}
\hline & \multicolumn{2}{c}{ Experimental group } & \multicolumn{2}{c}{ Control group } \\
\cline { 2 - 5 } Variables & Before & After & Before & After \\
\hline FMA-LL & $25.4 \pm 7.5$ & $27.4 \pm 6.7^{*}$ & $22.9 \pm 7.9$ & $25.9 \pm 4.8^{*}$ \\
BBS & $36.3 \pm 11.7$ & $46.7 \pm 9.4^{* *}$ & $39.0 \pm 8.7$ & $49.8 \pm 4.6^{* *}$ \\
6MWT (m) & $167.5 \pm 121.8$ & $261.0 \pm 115.4^{* *}$ & $157.5 \pm 64.0$ & $276.0 \pm 69.8^{* *}$ \\
K-MBI & $65.7 \pm 23.3$ & $87.0 \pm 10.5^{* *}$ & $57.4 \pm 22.4$ & $85.3 \pm 13.7^{* *}$ \\
\hline
\end{tabular}

Values are mean \pm standard deviation.

FMA-LL ${ }_{\text {paretic }}$ paretic lower limb score of the Fugl-Meyer assessment; BBS: Berg balance test; 6MWT: 6-minute walk test; K-MBI: Korean version of the Modified Barthel Index $* \mathrm{p}<0.05,{ }^{* *} \mathrm{p}<0.01$

Table 3. Changes in clinical outcome measures between pre- and post-treatment

\begin{tabular}{lcc}
\hline Variables & Experimental group & Control group \\
\hline$\Delta$ FMA-LL & $2.00 \pm 2.79$ & $3.00 \pm 4.60$ \\
$\Delta$ BBS & $10.40 \pm 8.25$ & $10.80 \pm 5.01$ \\
$\Delta 6$ MWT (m) & $93.50 \pm 56.28$ & $118.50 \pm 50.40$ \\
$\Delta$ K-MBI & $21.30 \pm 15.13$ & $27.90 \pm 14.93$ \\
\hline
\end{tabular}

Values are mean \pm standard deviation.

FMA-LL paretic $_{\text {: }}$ paretic lower limb score of the Fugl-Meyer assessment; BBS: Berg balance test; 6MWT: 6-minute walk test; K-MBI: Korean version of the Modified Barthel Index

basic ADL. These findings are consistent with those of a previous study in which circuit training therapy sessions with longer duration beneficially affected the effect on gait competency and balance function in chronic stroke patients ${ }^{4}$. To the best of our knowledge, few studies have investigated the effectiveness of circuit training in an inpatient rehabilitation setting for subacute stroke patients, and to compare the effectiveness of circuit training with individual physiotherapy in Korea.

Circuit training is a new model of rehabilitation service delivery and is distinct from the concept of group therapy, which is defined as therapy involving more than two patients, undertaking the same tasks at the same time under the direction of therapist with little or no individual tailoring or progression of activities ${ }^{5)}$. Circuit training comprises a set of core activities, individually adapted, task specific and graded in time ${ }^{14)}$. Its physiological approach is based on integrated functional exercises targeting specific impairments in strength, balance, endurance ${ }^{15)}$. A physiotherapist provides supervision and verbal feedback during circuit training; however, the majority of feedback is provided by setting up each task such that it provides a concrete goal and intrinsic feedback regarding its correct completion ${ }^{5)}$. For more appropriate participation in active rehabilitation practice in this study, we provided repetitive education and standardized guidelines were provided through a printed color manual and multimedia instruction on a set of core activities.

The mechanisms behind the circuit training eliciting similar degrees of improvement in lower-extremity motor control, balance, walking competency and basic ADL are uncertain, but there is evidence of a dose-response relation in the amount of early physical therapy after stroke and recovery of function ${ }^{16)}$. Several studies have proposed that although circuit training provide less opportunity for direct supervision and assistance, the total time spent in circuit training program is greater than in individual physical therapy, and that circuit training is highly effective at increasing the amount of time spent by subjects in active rehabilitation practice ${ }^{5,17)}$.

The findings of the present study are in line with the findings of English et al. ${ }^{5,17)}$ who also studied patients in an inpatient setting. Similar circuit-training protocols were used in several other trials. However, the circuit-training program in the present study can be discriminated for several reasons. (1) The intervention was performed by subacute stroke patients who were receiving inpatient rehabilitation. (2) The subjects of this study were able to perform 'sit-to-stand' activity, but required physical assistance or supervision by one person, while those of English et al. ${ }^{5}$ had the ability to walk a minimum of 10 meter without physical assistance. (3) In order to compensate for less opportunity of participation in active practice, we provide standardized therapy guidelines using a printed color manual and multimedia instruction on a set of core activities in addition to supervision and verbal feedback during the circuit training.

English et al. conducted a study of the amount of walking practice which was usually reported as 10-min duration in the previous studies ${ }^{16)}$, and confirmed that the duration of walking practice per circuit class therapy sessions was $10.5 \mathrm{~min}$, and that of individual physiotherapy sessions was $11.8 \mathrm{~min}^{17)}$.

The walking practice in our circuit training protocol was mainly distributed between categories 3 and 4 and a extent of treadmill or a stationary bicycle was used as an alternative method due to slow walking speed, poor balance control or safety issues. Thus, the duration of walking practice in our circuit training protocols lasted between 5 and 20 minutes depending on 
individual functional capacity.

The improvement in functional outcomes in the present study were comparable to those observed following circuit training by people who were living in the community ${ }^{7,14,15)}$. It was assumed that the activities of sitting with graded reaching tasks in category 1 would induce load on the hemiparetic lower limb eliciting motor recovery. Task specific practice, such as weight-bearing activities in category 2 is effective at promoting safe and independent mobility ${ }^{18)}$. The result suggest that activities with low functional level in our circuit training may be an appropriate substitution for practicing walking with maximal assistance by individual physiotherapy and they also indicate that circuit training might be appropriate for subacute stroke patients in both inpatient rehabilitation and outpatient settings. Inpatient rehabilitation services for stroke patients are increasingly limited by cost concerns ${ }^{5}$ as well as the current Korean healthcare environment. The circuit training program has the potential to provide longer therapy times with a lower staff-to-patient ratio, and it could be used as a cost effective strategy in rehabilitation services and as an alternative method to individual physiotherapy for stroke patients receiving inpatient rehabilitation.

There were several limitations to our study. First, the sample size was a relatively small, and subgroup analysis according to severity or type of stroke was not performed. Second, there was relatively short follow-up; therefore, the long-term effects of circuit training on walking competency after discharge remain unclear. Third, gait pattern or efficiency were not evaluated. The NDT adopts the current concepts as a problem solving approach to the assessment and treatment of individuals with disturbances of function, movement, and postural control due to a lesion of the central nervous system, and aims to identify and analyze problems individually within functional activities and participation in everyday life, as well as the analysis of movement components and underlying impairments ${ }^{13}$. Despite the standard circuit training guideline, our circuit protocol cannot be generalized to all stroke cases.

In conclusion, our circuit training program consisting of physical fitness training as well as mobility-related tasks with longer therapy time is feasible and had a high compliance rate among subacute stroke patients receiving inpatient rehabilitation who were at least able to stand independently. Thus, in the case of limited inpatient rehabilitation resource, circuit training therapy could be used as a cost-effective or alternative method to individual physiotherapy for improving lower-extremity motor control, balance, walking competency and basic ADL.

\section{ACKNOWLEDGEMENT}

This work was supported by the research grant of the Jeju National University in 2012.

\section{REFERENCES}

1) Chang WH, Kim YH: Robot-assisted therapy in stroke rehabilitation. J Stroke, 2013, 15: 174-181. [Medline] [CrossRef]

2) Werner RA, Kessler S: Effectiveness of an intensive outpatient rehabilitation program for postacute stroke patients. Am J Phys Med Rehabil, 1996, 75: 114-120. [Medline] [CrossRef]

3) Dean CM, Richards CL, Malouin F: Task-related circuit training improves performance of locomotor tasks in chronic stroke: a randomized, controlled pilot trial. Arch Phys Med Rehabil, 2000, 81: 409-417. [Medline] [CrossRef]

4) English C, Hillier S: Circuit class therapy for improving mobility after stroke: a systematic review. J Rehabil Med, 2011, 43: 565-571. [Medline] [CrossRef]

5) English CK, Hillier SL, Stiller KR, et al.: Circuit class therapy versus individual physiotherapy sessions during inpatient stroke rehabilitation: a controlled trial. Arch Phys Med Rehabil, 2007, 88: 955-963. [Medline] [CrossRef]

6) Holden MK, Gill KM, Magliozzi MR, et al.: Clinical gait assessment in the neurologically impaired. Reliability and meaningfulness. Phys Ther, 1984, 64: 35-40. [Medline]

7) Salbach NM, Mayo NE, Wood-Dauphinee S, et al.: A task-orientated intervention enhances walking distance and speed in the first year post stroke: a randomized controlled trial. Clin Rehabil, 2004, 18: 509-519. [Medline] [CrossRef]

8) Lee JJ, Lee HJ, Park JH, et al.: The korean version of berg balance scale as an index of activity related to ambulation in subjects with stroke. J Korean Acad Rehabil Med, 2007, 31: 400-403.

9) Kim H, Her J, Ko J, et al.: Reliability, concurrent validity, and responsiveness of the Fugl-Meyer assessment for hemiplegic patients. J Phys Ther Sci, 2012, 24: 893-899. [CrossRef]

10) Jung Y, Lee K, Shin S, et al.: Effects of a multifactorial fall prevention program on balance, gait, and fear of falling in post-stroke inpatients. J Phys Ther Sci, 2015, 27: 1865-1868. [Medline] [CrossRef]

11) Suzuki M, Fujisawa H, Machida Y, et al.: Relationship between the Berg Balance scale and static balance test in 
hemiplegic patients with stroke. J Phys Ther Sci, 2013, 25: 1043-1049. [Medline] [CrossRef]

12) Park JH: The effects of modified constraint-induced therapy combined with mental practice on patients with chronic stroke. J Phys Ther Sci, 2015, 27: 1585-1588. [Medline] [CrossRef]

13) Kollen BJ, Lennon S, Lyons B, et al.: The effectiveness of the Bobath concept in stroke rehabilitation: what is the evidence? Stroke, 2009, 40: e89-e97. [Medline] [CrossRef]

14) van de Port IG, Wevers L, Roelse H, et al.: Cost-effectiveness of a structured progressive task-oriented circuit class training programme to enhance walking competency after stroke: the protocol of the FIT-Stroke trial. BMC Neurol, 2009, 9: 43. [Medline] [CrossRef]

15) Duncan P, Studenski S, Richards L, et al.: Randomized clinical trial of therapeutic exercise in subacute stroke. Stroke, 2003, 34: 2173-2180. [Medline] [CrossRef]

16) Hillier S, English C, Crotty M, et al.: Circuit class or seven-day therapy for increasing intensity of rehabilitation after stroke: protocol of the CIRCIT trial. Int J Stroke, 2011, 6: 560-565. [Medline] [CrossRef]

17) English C, Hillier S, Kaur G, et al.: People with stroke spend more time in active task practice, but similar time in walking practice, when physiotherapy rehabilitation is provided in circuit classes compared to individual therapy sessions: an observational study. J Physiother, 2014, 60: 50-54. [Medline] [CrossRef]

18) Dean CM, Shepherd RB, Adams RD: Sitting balance II: reach direction and thigh support affect the contribution of the lower limbs when reaching beyond arm's length in sitting. Gait Posture, 1999, 10: 147-153. [Medline] [CrossRef] 